\title{
Der dunkle Fluss
}

Stefan Geil

By the rivers dark Where I could not see Who was waiting there

Who was hunting me.

- Leonard Cohen

Ich komme in das Zimmer und sehe graue Gesichter. Müde Augen blinzeln mir aus ihnen entgegen, während sich der Versuch eines Lächelns in Mundwinkel quält.

Die Luft ist abgestanden und der Geruch, den nur Krankenhäuser und Pflegeeinrichtungen in so unnachahmlicher Weise verströmen, betäubt mich beinahe. Sohn und Tochter schauen mit flehendem Blick zu mir. Zwischen ihnen im Bett liegt eine Person mit halbgeöffneten Augen.

Mit sichtlichen Problemen rasselt der viel zu langsame Atem durch die sterbensmüden Lungen der Mutter. Es klingt furchtbar. Bin ich noch ganz bei Trost, hier zu sein?

Ich bin Pfarrer. Und das ist mein Beruf.

Mit jedem einigermaßen festen Schritt komme ich näher, nehme in Windeseile alle Eindrücke in mich auf und weiß, was die Stunde geschlagen hat: Hier trifft jemand die letzten Vorkehrungen.

Hier gibt es keine Ausflüchte mehr. Hier geht es um's Sterben. In einer Minute oder einer Stunde. Vielleicht erst Morgen. Oder in paar Tagen. Aber es ist sicher, dass es geschehen wird.

Bald.

Ungeäußerte Wünsche schlagen mir in diesem verdichteten und stickigen Raum mit großer Gewalt entgegen. Manchmal habe ich das Gefühl, dass Angehörige meine Anwesenheit mit dem ersehnten Tod verknüpfen möchten. Der Pfarrer kommt, und deshalb kann der geliebte Mensch endlich gehen. Ich trage nie einen Talar bei solchen Besuchen, noch kenne ich jemanden, der das macht. 
Trotzdem verleihen mir Angehörige eine unbequeme Aura. Ich werde ungewollt zu Max von Sydow in Ingmar Bergmans Das siebente Siegel. Das schwarze Gewand denken sich die Menschen dann einfach mit. Ich werde zu jemanden gemacht, der für die allerletzten Dinge zuständig ist.

Ich habe gehört, dass in den USA bei Geiselnahmeverhandlungen kein Priester, Pfarrer oder Rabbi mit dem Geiselnehmer in Kontakt kommen darf.Aus dem einfachen Grund, dass der Geiselnehmer mit diesen Personen automatisch etwas Endgültiges und Abschließendes assoziieren könnte und sich so die Gefahr erhöht, dass er einen fatalistischen Weg einschlägt. Soweit zu Vorurteilen.

Auch hier scheint es unausgesprochen zwischen mir und den beiden Kindern im Raum zu stehen. Das Flehen in ihrem Blick ist auch das Flehen um ein Ende des Leids.

Die sichtliche Erschöpfung der beiden kennt keinen passenden Ausdruck mehr. Ihre Gesichter erzählen von langen Nachtwachen, völliger Müdigkeit und nagender Ohnmacht. Für einen Moment lähmt mich ihre Kraftlosigkeit. Dann geschieht etwas, das für mich - selbst im egoistischsten Sinne gedacht - ein kleines Wunder ist. Ihr Anblick hat meine Glieder schwer gemacht und meine Schultern nach unten gezogen.

Plötzlich nehme ich wahr, wie alles an mir leichter und meine Haltung fester wird. Das ist der Unterschied zwischen einem betroffenen Angehörigen und einem Menschen, der diese Szenerie betritt und sie später wieder verlassen kann. Der Unterschied zwischen privat und dienstlich.

Tochter und Sohn sind etwa in ihren Fünfzigern. Aber im Angesicht desVerlustes ihrer Mutter sagt das nicht besonders viel aus. Hier sind sie wieder Kinder, auch wenn sie es die letzten zwanzig oder dreißig Jahre vergessen haben sollten. Diese Situation erinnert sie seit unzähligen Stunden daran.

Ich biete den beiden an, einen Kaffee trinken zu gehen. Und ich verspreche ihnen, dass ich währenddessen ihrer Mutter nicht von der Seite weichen werde.

Es ist so, als wäre eine tonnenschwere Last von ihren Schultern genommen. Die Gesichter der beiden hellen sich auf, während ich die Hand der Mutter in meine nehme. Sie lag bis jetzt unter der Decke und ist schön warm. Die beiden verabschieden sich und gehen mit unsicheren Schritten aus dem Zimmer. Jetzt bin ich mit der Frau allein.

Es ist schwer zu reden und laut zu beten, während ihre gedehnten und ungesund klingenden Atemzüge alles zerhacken. Diesen Ge- 
danken bereue ich sofort. Ich bin hier, um für jemanden da zu sein. Und ich fange trotzdem an, die Atmosphäre zu bewerten, als wäre ich im Gottesdienst und werde von zu lauten Konfirmanden gestört. Ist das pietätlos? Vermutlich. Nein, ganz sicher, ermahne ich mich selbst. Was lernt man an der Seite eines sterbenden Menschen?

Grenzen des eigenen Selbst und zwar deutlich. Ich habe genug von mir, konzentriere mich auf die gurgelnden, rasselnden Geräusche und auf die Person neben mir im Bett.

Eigentlich sieht sie mit ihrem runden Gesicht und den wie schläfrig wirkenden, halboffenen Augen gar nicht so schlecht aus. Kaum eine Falte steht auf ihrem vollen Gesicht. Keine Spur von tiefen Furchen oder Ausmergelung. Aber ihre mühsame Atmung singt ein ganz anderes Lied.

Sie muss einmal eine sehr hübsche Frau gewesen sein, denke ich bei mir.

Ich spreche mit ihr. Stelle mich ihr vor. Erzähle ihr, wo ich herkomme und dass ich jetzt eine Weile bei ihr sein werde. Lege ein Holzkreuz mit fein geschliffenen und abgerundeten Kanten in ihre Hand und beginne langsam Psalm 23 zu beten. Sie zeigt keine Reaktionen darauf. Aber ich bin mir fast sicher, dass sie mich hören kann und auch die Berührung wahrnimmt ...

In meinem ersten Amtsjahr habe ich einunddreißig Menschen beerdigt. Es war technisch gesehen kein Problem, die Angehörigen zu begleiten und die Trauerfeiern zu gestalten. Probleme haben mir ganz andere Dinge gemacht.

Es hat mich furchtbar erschreckt, dass ich mich nach dem zwanzigsten Sterbefall kaum noch an den zweiten, dritten und vierten erinnern konnte. Den Angehörigen geht es da zwangsläufig anders: der Unterschied zwischen privat und dienstlich.

Aufmerksam habe ich den Berichten vom Sterben der geliebten Menschen, der Ehemänner, Ehefrauen, Schwestern und Brüder oder der Kinder zugehört. Das ist Teil meines Berufes, und ich bin darauf angewiesen, so etwas zu wissen. Nicht nur für die Ansprache bei der Trauerfeier, auch um später Irritationen zu vermeiden. Nichts kann schlimmer sein als am Grab eines Angehörigen oder Freunds $\mathrm{zu}$ stehen und zu hören, dass er jetzt friedlich entschlafen sei, wenn man weiß, dass es ganz gewiss nicht so passiert ist. Dann wandelt sich jeder Zuspruch in blanken Hohn. Ich bin es den Toten schuldig, mir das anzuhören und in angemessener Form zu berücksichtigen.

Neben dieser rein professionellen Aufmerksamkeit muss ich gestehen, dass mich auch ein ganz persönliches Interesse genau hinhören lässt. 
Beim Lauschen dieser teils sehr unangenehmen - da detaillierten - Berichte der Hinterbliebenen wird mir wieder aufs Neue klar, was mir selbst als Erfahrung fehlt.

Es hat seinen Schrecken immer noch nicht verloren, dass ich niemals einen Menschen hab' sterben sehen. Obwohl ich so oft mit diesem Thema beruflich und privat konfrontiert wurde.

Jedes Mal war ich zu früh dran. Oder zu spät.

Das Paradoxe daran ist, dass ich mir keinesfalls wünsche mitzuerleben, wie ein Mensch stirbt.

Ich fürchte mich davor. Aber ich kann dieser Furcht nur auf eine Art begegnen: indem ich mich darauf vorbereite, ihr zu begegnen. Angstlust nennt man das wohl. Genauso, wie man sich überwindet, einen sehr unangenehmen Gruselfilm bis zum Schluss zu schauen. Nur um zu sehen, was von ihm im Kopf bleibt. Ob sich noch nach Tagen die Hautporen um jedes Härchen festziehen und um zu sehen, wie man damit fertig wird.

Eine weitere Facette meiner Beerdigungspraxis - der Angstlust gar nicht so unähnlich - ist der Rausch. Das klingt möglicherweise bedenklich, aber ich empfinde es so: Ich fühle mich an keinem Ort lebendiger als in der Gegenwart eines Toten. Ich glaube, wer regelmäßig an offene Gräber tritt, wird mit der geballten Kraft des Gefühls, am Leben zu sein, getroffen. Das ist - in Ermangelung eines besseren Ausdrucks - wie ein Rausch.

Ich habe oft wahrgenommen, dass mir nach einer gelungenen Trauerfeier die schwierigsten Aufgaben leichter von der Hand gehen. Und ich möchte wetten, dass das unter anderen Umständen sicher nicht so wäre. Meine Lebendigkeit trägt mich eine Zeit lang traumtänzerisch durch meine anderen Aufgaben. Mein Serotoninspiegel sieht dann bestimmt rosig aus. Obwohl ich dem Gefühl nach nicht mehr tue, als eigene Ängste wohldosiert zu durchleben. Für Augenblicke klart sich auf, was die meiste Zeit über verborgen bleiben muss: Mein eigenes Am-Leben-Sein ist nur ein vorübergehender Zustand. Und meine Beziehung zur Welt bestenfalls zeitweilig. Die flüchtige Bekanntschaft einer Fremden, die sich möglicherweise gar nicht großartig ändert. Nur das Staunen über sie.

Nach getaner Arbeit überlagert sich dieser Gedanke wundersam mit allen Formen der Unterhaltung: Nicht heute! scheinen diese Ablenkungen mir hysterisch entgegen zu rufen: Ich werde heute nicht sterben!

Während eines kurzen Praktikums in einer Lungenklinik hatte ich mit vielen COPD-kranken Menschen zu tun. Kaum ein Ort hat mir so plastisch unsere Zerbrechlichkeit vor Augen geführt. Mit jedem 
qualvollen und kraftlosen Luftschnappen, das ich wahrgenommen habe, wuchs in mir der Wunsch, eine Zigarette zu rauchen. Bis zum Ende des Tages habe ich damit gewartet. Als ich endlich da angekommen war, schien sich alles, was mir an diesem Tag schwer auf der Seele lag, zu verflüchtigen. Gemeinsam mit dem bläulichen Qualm. Magisch zieht der Rauch seine Schlieren vom Hier und Jetzt in ein unsichtbares Nirgendwo.Viele halten es für ein Zeichen der Lebensbejahung, aktiv zur Zerstörung seiner Lungen beizutragen. Es ist gar nicht so ungewöhnlich, dass Menschen, die regelmäßig mit dem Tod zu tun haben, rauchen. Das Lebendige daran ist die Möglichkeit, weiterhin etwas Falsches machen zu können und diese Möglichkeit wahrzunehmen. Gerade im lustvollen Begehen von kleinen Fehlern steckt eine Sterbensseele.

Die Endlichkeit meiner Handlungen in meinen Horizont hineinzulassen, mich damit auseinanderzusetzen und über den Tod nachzudenken. Das ist für mich wie einen dunklen Fluss entlang zu gehen. Ein Bild, das in mir sowohl Unbehagen als auch Neugier weckt.

Unbehagen, weil ich nicht weiß, was darin zu finden ist. Neugier, weil meine Spekulationen darüber beinahe kein Ende nehmen. Jeder feste Schritt am Ufer dieses Flusses bringt neue Kraft in die Frage seines Inhalts. Das trübe Wasser lädt die Phantasie ein.

Wird sich etwas ändern, wenn ich weiß, was in diesem Fluss ist? Wenn ich endlich einen Blick hineinwerfen und durch das Wasser bis auf den Grund sehen kann. Werde ich mich ändern?

Von dieser Frage scheint mir eine andere stark abhängig zu sein: Warum mache ich das überhaupt? Was springt für mich dabei raus?

Einfach gesagt: das Wunder.

Das Wunder ist für mich zu gleichen Teilen Traumabewältigung, außergewöhnliche Erfahrung und außerordentlicher Bewegungsspielraum. Alles drei in einem.

Jemand hat mir einmal den schlichten, aber klugen Satz auf den Weg gegeben: Immer der Angst nach. Und ich bin trotz gegenteiliger Meinungen ein eher ängstlicher Mensch. Was mich nicht davon abhält, mich mit allem, was mir Angst macht, auseinanderzusetzen.

Das Wunder ist für mich: etwas von seiner Arbeit zu verstehen, die schmutzig sein kann und einen mit Schrecklichem in Berührung bringt. Aber auch aus einem großen Schatz an außergewöhnlichen Erfahrungen zu schöpfen. Ganz gleich, ob sie guter oder schlechter Natur sind.

Immer wieder höre ich den Satz: "Ich mache das, um den Menschen $\mathrm{zu}$ helfen."Ich bewundere es, wenn andere das über ihre 
Arbeit sagen können. Bei mir ist es anders. Ich mache das in erster Linie, um mir zu helfen.

Der Sinn, den ich in meinen privaten Verlusten gefunden habe, ist dieVertrautheit mit Verlusten, die ich sinnvoll nutze. Die erste Leiche, die ich gesehen habe, war die eines geliebten Menschen.

Es war wie ein Erwachen. Und mit ihm kam Erkenntnis.

Manchmal passiert das ganz schrittweise. Manchmal plötzlich. Einige von uns werden im Kindesalter behutsam herangeführt. Mit unserem ersten Haustier, das eines Morgens reglos daliegt. Oder mit einer Ameise, die sich, nachdem wir mit ihr gespielt haben, nicht mehr rührt.

Schritt für Schritt wird es uns bewusst:

egal, ob wir uns Christen, Juden oder Muslime nennen; egal, welche Weltanschauung wir haben.

Wir sind alle Teil der größten Gemeinde, die es auf der Welt gibt. Es ist die Gemeinde der Lebenden.

Es gibt kein Gesetz, das uns die Mitgliedschaft in der Gemeinde der Lebenden unbedingt zusichert.

Es ist eher so, als würde ein launischer Rausschmeißer einen heute kommentarlos durchwinken, aber am nächsten Tag den Zugang auf ewig versperren.

Die Gesetzlosigkeit, mit der sich Leben verliert, ist eigentlich der Stoff, aus dem die wahren Alpträume gemacht sind.

Das ist ein schrecklicher Gedanke, der hilflos macht und Verzweiflung schürt. Jeder entwickelt seine eigene Strategie, um mit ihm klar zu kommen. Ich habe mich dafür entschieden, ihm zu begegnen. Ich mache mich jedes Mal, wenn ich bei Angehörigen bin, ein kleines bisschen mehr mit ihm vertraut. Und gleichzeitig habe ich ihm den Krieg erklärt.

Meine Vertrautheit mit den Empfindungen während eines Verlustes sind die besten Voraussetzungen, um in dem Bereich zu arbeiten. Das ist einem trockenen Alkoholiker, der ein Treffen der Anonymen Alkoholiker moderiert, gar nicht mal so unähnlich.

Außerdem ist da für mich noch dieser Traum vom guten Sterben: dem oft zitierten "alt und lebenssatt" werden. Oder dem bewusst erlebten und gestalteten Ende. Diesen Traum finde ich an den ungewöhnlichsten Orten. In den Büchern und Filmen, die ich verschlinge, als würde der Tod schon hinter mir stehen.

Ein durch und durch fatalistischer Film wie Pat Garrett \& Billy the Kid aus dem Jahre 1973 leistet sich für eine Nebenfigur eine wunderschöne Sterbeszene. 
Für diese Sequenz wurde das Bob-Dylan-Lied »Knocking on Heavens Door eigens geschrieben.

Die Idee zu der Szene muss der Regisseur Sam Peckinpah tief auf dem Grund einer Whiskeyflasche oder in den unteren Reihen eines Pillenröhrchens gefunden haben.

Alkohol und Drogen waren Peckinpahs Weg, diese Welt zu verlassen. Im Rausch soll er einmal mit einem langläufigen Revolver auf sein Spiegelbild geschossen haben. Diese verzweifelte Mach-kaputt-was-dich-kaputt-macht-Handlung hat Peckinpah sogar in das Filmfinale integriert. Das spricht nicht unbedingt für ein gesundes Verhältnis von Leben und Sterben in Peckinpahs Geist.

Und trotzdem ist da diese eine Szene, die scheinbar aus dem Herzen eines zutiefst mit dem Tod versöhnten Menschen kommt.

In besagter Szene macht die Handlung, die eigentlich von den beiden Ikonen Pat Garrett und Billy the Kid erzählt, einen Schlenker zu der Nebenfigur Sheriff Baker. Über ihn lernt man nicht viel. Nur, dass er in die Jahre gekommen ist und lieber zuhause an seinem Boot herumtischlert, als aufVerbrecherjagd zu gehen. Mit dem Boot möchte er endlich über den Fluss aus seiner armseligen Stadt abhauen. Aber gemeinsam mit seiner flintenbewährten Frau, die er nur Momma nennt, zieht er noch einmal mit Pat Garrett los.

Bei einer Schießerei wird Baker jedoch tödlich getroffen. Leise wird während dieser Sequenz "Knocking on Heavens Door eingespielt. Das Lied wächst in die Szenerie hinein, in der natürlich gerade die Dämmerung anbricht, so dass jeder Maler der Romantik seine wahre Freude daran gehabt hätte.

Baker schleppt sich mit letzter Kraft an den Fluss, seine Frau kommt zögernd hinter ihm her. Erst lächelt sie, dann beginnt sie ungläubig zu weinen. Baker schaut über den dunklen Fluss und sein Blick schweift in die Ferne. Obwohl man Bakers eigentlichen Tod nicht sieht, ist das für mich der Gänsehautmoment des Films. Vielleicht, weil eine im Grunde unwichtige Figur mit einer derartigen Würde behandelt wird, während die beiden Hauptfiguren relativ unspektakulär weggeputzt werden.

Bittersüß und den Film seltsam brechend ist diese kurze Episode. Der Regisseur hatte die Idee, dass die gesamte Geschichte, die er erzählt, nur eine kurze Vision in der Sterbesekunde Pat Garretts ist. Und trotzdem ist da dieser Fitzel vom guten Tod bewahrt, der mich immer wieder berührt. Möglicherweise aus einem ganz einfachen Grund: Ich kann mir bessere Welten vorstellen. Oder andere. Aber ich kann mir nicht vorstellen, meine eigene zurückzulassen. Wenn ich über die Figur von Baker nachdenke, dann rückt mein eigenes 
Ableben wieder merklich nach hinten, in mein hohes Alter. Aber es kann an einfach jeder Stelle meines Lebens unverhofft in meine Pläne hereinbrechen. Baker ist für mich die Projektionsfläche dieser Gedankenwindung, die mich ergreift und traurig werden lässt.

Sheriff Baker will sowieso weg und sehen, was jenseits des dunklen Flusses liegt. An dem Boot baut er schon emsig. Nur tritt er letzten Endes schneller und anders als erwartet diese Reise an. In meinen Überlegungen über meinen eigenen Tod merke ich, dass sie viel stärker von Träumen und Vorstellungen als von meinerVernunft befeuert sind. Da, wo alle Sinne in einen Reigen miteinander treten. In meinen Träumen finde ich oft markante Bilder, die mir wie Erklärungsversuche meines Unterbewusstseins vorkommen.

Eines Nachts habe ich Folgendes geträumt. Ich war wieder an alten Orten, die ich im noch recht kurzen Leben zurück gelassen habe. Erinnern kann ich mich später nach dem Aufwachen nur noch an Heidelberg, wo ich einen Teil meines Studiums verbracht habe.

Mehrere Jahre später bin ich ein paar Mal da gewesen. Nur um festzustellen, dass die Stadt, wie ich sie damals kennen gelernt habe, jetzt nicht mehr da ist.

Zum Beispiel das ABM-Wohnheim im Pfaffengrund, in dem ich gewohnt habe, weil ich am Anfang nichts in der Innenstadt gefunden hatte.

Es war ein nigelnagelneuer Bau - krankenhausartig, alles in weiß und creme. Nur sechs Jahre später ist das weitläufige Gebäude komplett abgerissen worden.

Ich wette, es hat immer noch diesen Geruch von frischer Farbe und Möbellack versprüht, als es dem Erdboden gleich gemacht wurde.

Auf der Fläche steht jetzt ein Kinderspielplatz. Neues musste noch Neuerem weichen. Die Entropie erstreckt sich nicht nur auf unsere Körper und Geister.

Auch der Platz, den ich immer wieder mit einem Sack nasser Wäsche überqueren musste, um zum Waschsalon um die Ecke zu gelangen, wurde vollständig planiert. Wie im Tagebau hat man ein tiefes Loch ausgehoben, um dort ein Parkhaus für die vollkommen überfüllte Altstadt unterzubringen. Gelegen an der Straße mit dem wunderbaren Namen Plöck: nach der Grenze zwischen Nord- und Südkorea, die wohl tödlichste Stelle auf dem Planeten. Vor allen Dingen, wenn man Radfahrer ist.

Im Traum ist alles wieder da, wo es sein sollte bzw. so, wie ich es damals kennengelernt habe. 
Eine mürrische zeitlos aussehende Frau führt mich herum, an alle Orte, die ich vermisse.

Vermisse, weil sie zwar noch da sind, aber nicht mehr so, wie ich sie erlebt habe. Irgendwie habe ich das Gefühl, die Frau unbestimmbaren Alters arbeite nur halbtags in dem Job, den sie gerade mit mir erfüllt. Und es ist die schlechtere Hälfte.

Wie ein lustloser Fremdenführer sagt sie in leicht schwäbischen Singsang: "Hier« oder "Schau nur, da«, während wir in Windeseile überall vorbeizischen.

Zurück in ihrem WG-Zimmer werde ich ausgefragt. Warum ich hier sei? Warum ich so dick geworden sei? Warum ich nicht mehr Sport mache? Was ich mit meinem Leben anfangen wolle?

Etwas Verbissenes liegt in ihrer Stimme.Verbissen und gleichzeitig kraftlos. Ich denke bei mir: Junge Dame! Entweder du reißt dich jetzt zusammen, oder du nimmst eine Lexotanil aus der Packung, die du bestimmt im Bezug deines Kopfkissens versteckt hast.

Doch bevor es dazu kommt, wird sie von einer neuen Reisebegleiterin abgelöst. Eine sehr alte und sehr beleibte, kleine Dame nimmt mich an der Hand, wie ein Kindergartenkind.

Es geht durch runde Kellertüren immer weiter die Treppe hinunter. Bis mir kaltes Wasser bis zu den Knien reicht und alles in ungesunden grünen Schein getaucht ist. Hier bin ich ebenfalls schon gewesen. Auch wenn ich mir sicher bin, dass das hier nicht wirklich ein Ort ist. Kein Ort, sondern ein Zustand.

Wie ein zäher Teig legt sich die Schwere über jedes meiner Glieder. Meine traurigsten und extremsten Erinnerungen sind hier untergebracht. Eine Welt der Traumata vieler Zeiten und vieler Orte. Stimmungsvoll arrangiert für mich.

Die alte Dame führt mich ausnehmend freundlich herum. Sie beschreibt alles genau, obwohl ich eigentlich weiß, was hier alles zu finden ist.

Sie lässt dabei meine Hand niemals los. Die ist das Einzige, was sich hier unten warm anfühlt. Nicht gerade viel, woran man sich wärmen kann. Aber mir reicht es.

Wieder findet ein Reisebegleiterwechsel statt.

Ein hagerer alter Mann, der offensichtlich blind ist, bringt mich in einen hässlichen Plattenbau. Ich habe keinen Schimmer, wo dieses Hier sein soll.Vollkommen ohne Orientierung blicke ich mich um und sehe nur blassen Beton und freiliegende Treppen. Sie führen nirgendwo hin, und ihre Anordnung macht einfach keinen Sinn.

Ein kleines Kind hätte das entwerfen können, wenn es einen Gebäudeplan zerrissen und die Teile einfach in die Höhe geworfen 
hätte. Immer panischer sehe ich mich um. Der blinde Mann beginnt leise zu summen.

Ich merke, dass mein Traum mir immer weiter auseinanderfranst. Wie eine alte Decke, die sich in ihre Bestandteile auflöst. Schläfrig, irgendwo in einer nebligen Ecke meines Geistes, nehme ich das wahr, während mein Aufwachen einsetzt. Das Gefühl, hier weg zu müssen und all die Verwunderung zurückzulassen, wird durch die Klarheit des Erwachens schließlich überholt. Ich öffne die Augen, und langsam gewinnt eine andere Welt helle Konturen.

Vielleicht könnte so mein eigenes Sterben aussehen, denke ich mir später. Ein mehr oder weniger sanftes Geführtwerden: an Orte, die ich kannte und die ich vermisse. Ebenso dahin zurück, wo ich nicht gerne gewesen bin. Nur um zu sehen, dass es nicht so schlimm ist, wenn du eine warme Hand gereicht bekommst, an der du dich festhalten kannst. Ich darf mir alles noch einmal ansehen.

Bis ich damit fertig bin, alles gesehen habe und mich jetzt weiter führen lassen kann. $\mathrm{Zu}$ einem Ort, der mich ratlos macht und durcheinander bringt. Jede meiner Erwartungen unterwandert. Vielleicht wie ein plötzliches Aufwachen aus dem, was mir eben noch wie ein festes Fundament - eine unumstößliche Wahrheit - vorgekommen ist.

Oder es ist alles nur eine sinnlose Aneinanderreihung von sich vermischenden Eindrücken. Die letzten Fünkchen des Leuchtfeuers meiner in Not geratenen Neuronen, bevor das Nichts, die Schwärze kommt.

Es ist absurd, dass mein Traum in jedem zu erwartendem Fall für mich eine vollkommen verständliche Darstellung des Sterbens ist. Denn ich bin mir sicher: Das gilt einfach für jedeVorstellung, die ich vom Sterben habe. Sie fungiert als perfekter und zugleich ernsthafter Zeitvertreib. Bloße Unterhaltung.

Und sie wird trotzdem spätestens dann obsolet, wenn ich mit dem Sterben beginne ...

Zurück.

Ich bin wieder bei der Frau mit der schweren Atmung. Ich war jetzt fast 90 Minuten hier und muss weiter. Zum nächsten Termin.

Ich bete mit den beiden zurückgekehrten Kindern und verabschiede mich.

Auch dieses Mal lebt der Mensch noch, den ich ein kurzes Stück weit begleitet habe. 
Vielleicht wird sich wie in meinem Traum jetzt jemand anderes um die Reisebegleitung kümmern. Eine warme Hand in einem weiteren dunklen und dreckigen Kellerloch, durch das jemand durch muss.Vielleicht.

Vieles leicht nehmen und so versuchen, Schweres zu erörtern. Dem dunklen Fluss folgen, um zu sehen, wohin er fließt. Das werde ich machen.

Ich werde weiterhin einer Welt voller schöner und schrecklicher Möglichkeiten begegnen und über ihr zu einem Schluss kommen. Das ist das Kerngeschäft. Und wenn es gelingt - ein Wunder.

Ich bin Pfarrer. Und das ist mein Beruf.

— Stefan Geil ist evangelischer Theologe und arbeitet als Pfarrer in der Lukasgemeinde in Lampertheim. Zudem wirkt er als Mitglied der Jury der Evangelischen Filmarbeit und ehrenamtlicher Prüfer der Freiwilligen Selbstkontrolle der Filmwirtschaft (FSK). 